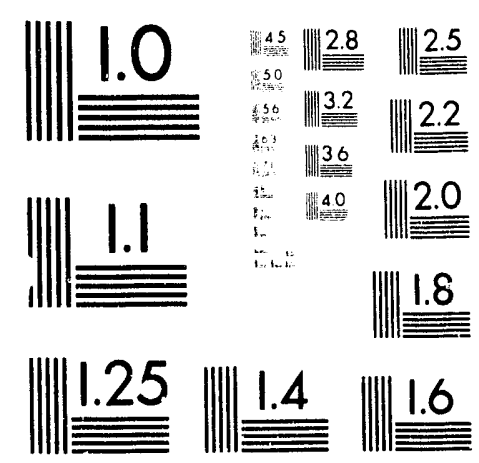



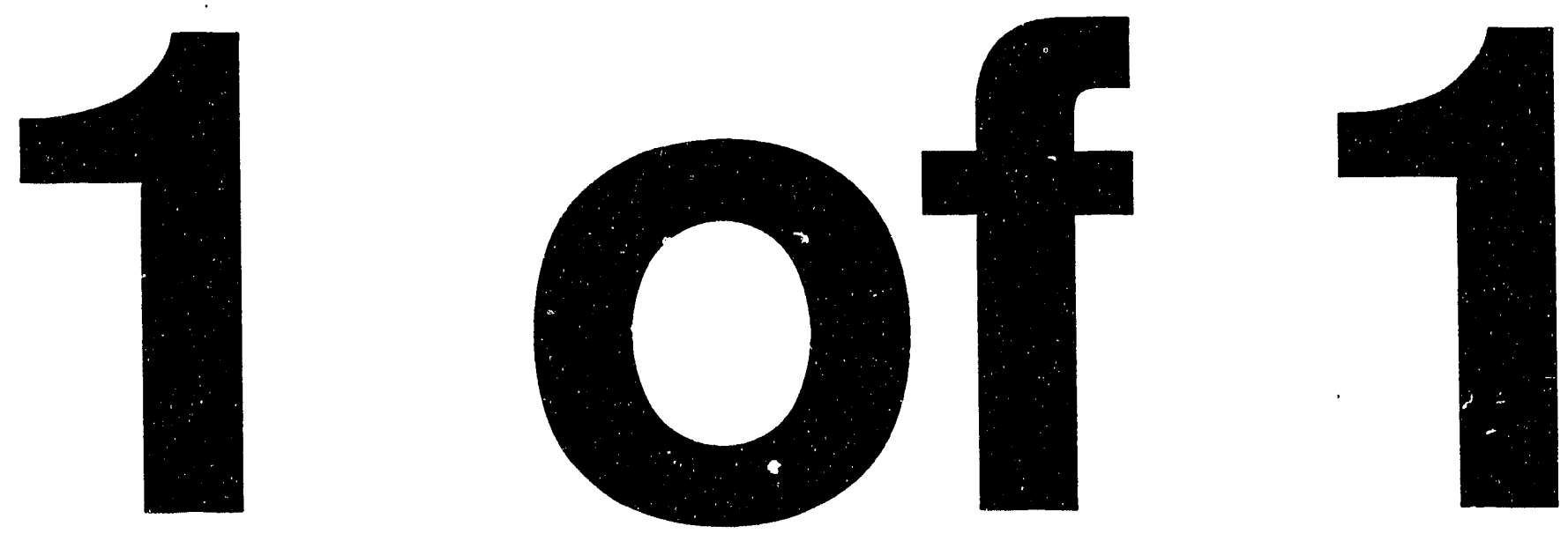


\title{
Conf-93c6.245-1
}

UCRL-JC-114438

PREPRINT

\section{Sodium-Layer Laser Guide Stars}

\author{
Herbert W. Friedman
}

This paper was prepared for submittal to the NATO/ASI

Conference on Adaptive Optics for Astronomy held in Cargese, France on June 29 thru August 9, 1993

KUE CEV

August 3, 1993

AUG 301993

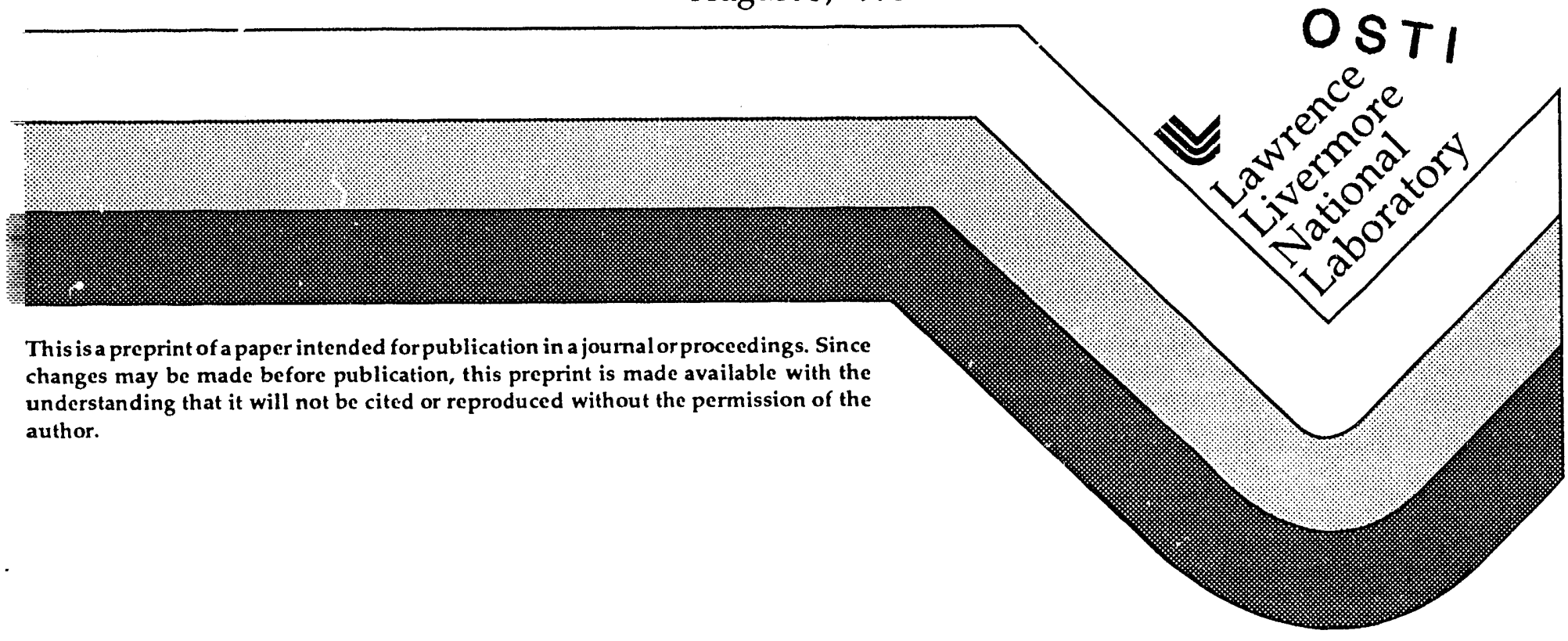




\section{DISCLAIMER}

This document was prepared as an account of work sponsored by an agency of the United States Government. Neither the United States Government nor the University of California nor any of their employees, makes any warranty, express or implied, or assumes any legal liability or responsibility for the accuracy, completeness, or usefulness of any information, apparatus, product, or process disclosed, or represents that its use would not infringe privately owned rights. Reference herein to any specific commercial products, process, or service by trade name, trademark, manufacturer, or otherwise, does not necessarily constitute or imply its endorsement, recommendation, or favoring by the United States Government or the University of Californla. The views and opinions of authors expressed herein do not necessarily state or reflect those of the United States Government or the University of California, and shall not be used for advertising or product endorsement purposes. 


\title{
SODIUM-I A YER LASER GUIDE STARS
}

\author{
H. W. FRIEDMAN \\ Lawrence Livermore National Laboratory \\ P. O. Box $808, L-464$ \\ Livermore, CA 94551
}

\begin{abstract}
The requirements and design of a laser system to generate a sodium-layer beacon is presented. Early results of photometry and wavefront sensing are given.
\end{abstract}

\section{Introduction}

Sodium-layer laser guide stars 1,2 have the potential to allow adaptive optics systems to accomplish highorder wavefront corrections of atmospheric turbulence even for the largest ground-based telescopes. We have initiated a program whose long-term goal is to demonstrate the feasibility of closed-loop adaptive optics with sodium laser guide stars, using existing lasers at LL.NL developed for the Department of Energy's Atomic Vapor Laser Isotope Separation (AVLIS) program. These copper-vapor-laser pumped dye lasers are considerably larger and more powerful than will be ultimately needed at an observatory site, but they have several immediate advantages for a feasibility experiment: they are available now, they have been engineered for high reliability, and the dye lasers are highly tunable and controllable in terms of line shape.

\section{System Concept}

Adaptive optics systems using laser guide stars must filter out the Rayleigh scattered light from the lower atmosphere. This can be accomplished by either temporal gating or spatial discrimination. In the temporal gating method, the pulse repetition frequency must be about $1 \mathrm{kHz}$ which excludes many interesting laser configurations. We have chosen the spatial discrimination method in which the laser is propagated from a separate beam director and a field stop in the optical train then rejects the lower-altitude Rayleigh scattered light, as shown in Fig. 1.

Measurements at our site suggest that the parameter, $r_{0}$, is approximately $5 \mathrm{~cm}$ at a wavelength of $0.5 \mu$. For our half-meter telescope, the parameter $\mathrm{D} / \mathrm{r}_{\mathrm{O}} \approx 10$ at a wavelength $\lambda=0.5 \mathrm{~m}$, dropping to $\mathrm{D} / \mathrm{r}_{\mathrm{O}}=$ 6 at a wavelength $\lambda=0.8 \mu$. Based on these values, a zonal adaptive optics system optimized for operation at a wavelength of $\lambda=0.8 \mu$ would have $9 \mathrm{~cm}$ subapertures, so that our half-meter telescope would require about 20 subapertures in all. 


\section{Site Layout}

The Laser Guide Star site is located approximately $100 \mathrm{~m}$ from the laser building. The dye laser beam is propagated through an underground, evacuated pipe to a vacuum chamber directly under the guide star site where a pointing and centering control loop, beam diagnostics, communication equipment and safety shutters are located. The final mirror turns the beam upwards into a vertical beam tube. At the top of the beam tube is an exit window which is the final vacuum interface into the atmosphere. The laser beam shape is rectangular with dimensions of $4 \mathrm{~cm} \times 8 \mathrm{~cm}$, commensurate with a value of $\mathrm{r}_{\mathrm{O}} \approx 5 \mathrm{~cm}$ at LLNL. At present the beam propagates to zenith with no positioning or tracking capability, as shown in Fig. 2. The experiments we report on here concern creating the guide star, performing imaging and photometry measurements on it, and recording open-loop wavefronts. A beam director was installed in the summer of 1993 but no results are available at this time.

\section{Laser Requirements}

The laser power required to produce a sodium beacon bright enough to close a control loop at the telescope depends upon the parameters of the sodium laser and upon the cumponents of the control system, e.g., telescope optical efficiency, sensor quantum efficiency and noise characteristics. The maximum emission rate for a column of sodium atoms, assuming an illuminating laser pulse duration longer than the spontaneous lifetime (CW limit) is given by the cclumn density divided by the emission lifetime. The

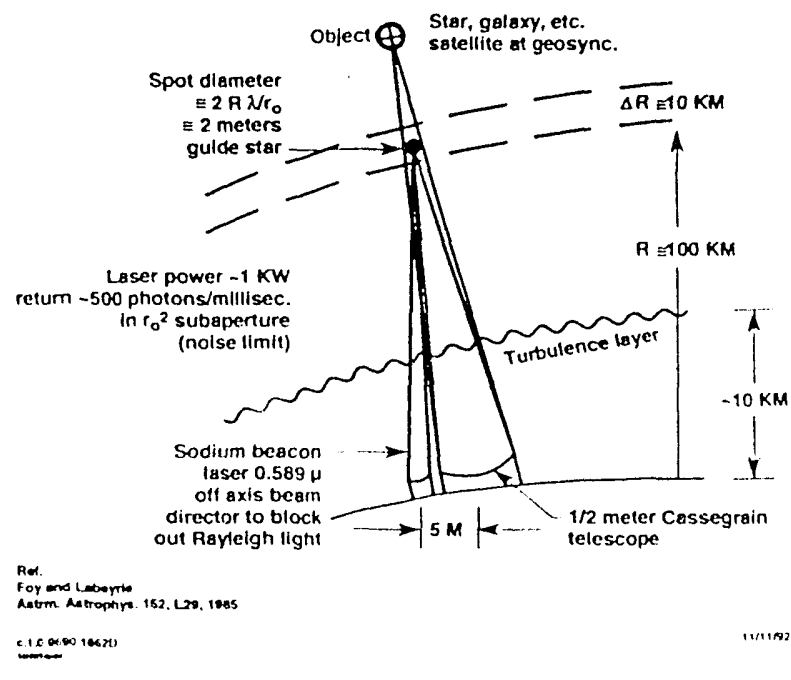

Fin- 1. Laser and beam-director configuration.

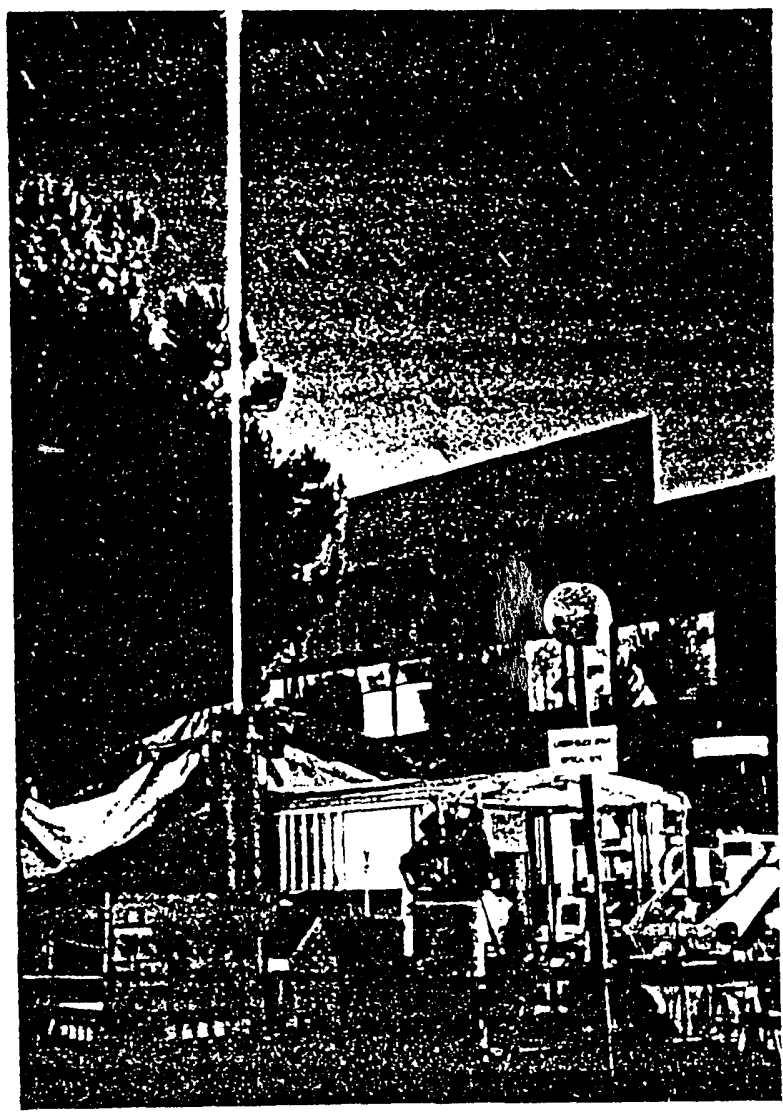

Fin. 2. LINL I aser Guide Star sile wilh 1100 W sodium hesaenlasin 
Table 1: Properties of the atmospheric sodium layer and the LLNL site.

\section{Atomic Parameters}

Sodium D2 line wavelength

Spontaneous emission lifetime

Degeneracy correction factor

\section{Atmosphieric Sodium Layer Parameters \\ Mean coluinn density at zenith (annual variation of factor of two)}

Mean altitude

Vertical depth

Optical depth at zenith ( $3 \mathrm{GHz}$ bandwidth)

Emission cross section

(averaged over $3 \mathrm{GHz}$ bandwidth)

Atmospheric Propagation Parameters (for LLNL site)

Transmission coefficient, ground to sodium lay'er

Turbu'ence coherence length, at $500 \mathrm{~nm}$ (typicai)

Illumirated spot area at the $\mathrm{Na}$ layer

Radiating spot area at the $\mathrm{Na}$ layer*

Wavefront Sensor (LLNL experiment)

Subaperture area

$\begin{array}{ll}\lambda \mathrm{Na} & 589 \mathrm{~nm} \\ \tau_{\mathrm{n}} & 16 \mathrm{~ns} \\ \mathrm{gu} /\left(\mathrm{gl}+\mathrm{gu}_{\mathrm{u}}\right) & 0.67\end{array}$

$\mathrm{N}$

$5 \times 10^{13} \mathrm{~m}^{-2}$

$\mathrm{z} \quad 95 \mathrm{~km}$

$\Delta \mathrm{z} \quad 10 \mathrm{~km}$

$2.5 \%$

$\sigma \quad 4 \times 10^{-12} \mathrm{~cm}^{2}$

* For saturated sodium emission, $A_{\text {rad }}$ is larger than $A_{S}$ because the wings as well as the core of the illuminating profile begin to saturate.

photon rate, $\mathrm{dN} \mathrm{ph}_{\mathrm{pt}} / \mathrm{dt}$, at a detector on the ground with area equal to the wavefront sensor sub-aperture area $A_{w f s}$, is then given by:

$$
d N_{p h} / d t \leq\left(A_{\text {rad }} N / \tau_{n}\right)\left(g_{u} / g l+g_{u}\right)\left(A_{w f s} / 4 \pi z^{2}\right) f_{d} X
$$

where $f_{d}$ is the duty factor of the laser and the rest of the symbols are defined in Table 1. For the values quoted in Table 1 , typical of the LLNL site, the maximum photon rate at the ground based detector is $\mathrm{dN}_{\mathrm{ph}}$ $/ \mathrm{dt} \leq 3.6 \times 10^{8} \mathrm{f}_{\mathrm{d}}$ photons per second per subaperture.

The minimum number of photons per integration time needed to close a control loop is a function of the detector quantum efficiency and the noise floor. For a typical intensified fast-framing camera with quantum efficiency of about $5 \%$, the minimum number of photons needed for high performance is of the order of one or wo thousand. (Bare CCD's with high quantum efficiency would need far fewer photons.) For a typical atmospheric change frequency of $100 \mathrm{~Hz}$, and a wavefrom sampling rate of $10 \times$ that or $1 \mathrm{kHz}$, the integration time is of the wider of a millisecond. Assuming an optical irain efficiency of $30 \%$, the laser duty factor, $f_{d}$, must be at least $2 \%$.

For the experiments described in this paper, the AVLIS laser's duty factor $f_{d}$ is about 10-3. Hence the flux of photons per second will be lower by the corresponding ratio. These lower photon fluxes can be utilized for closed-loop operation if the integration time is made fonger than one millisecond, as would pertain to atmospheric conditions charactized hy lower valuesof the atmospheric chance frequency and

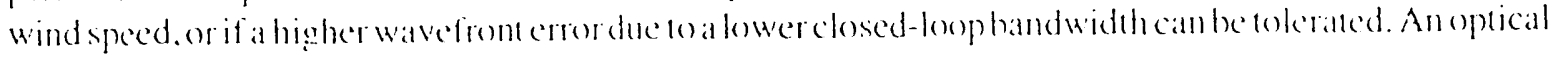


pulse stretcher has been built to extend the duty factor by a factor of 16 . This pulse stretcher will be used in upcoming experiments to close the control loop with $1 \mathrm{~ms}$ integration time.

The average laser power is determined by the laser flux required to generate approximately 1000 photons in the subaperture in the integration time of $1 \mathrm{~ms}$, i.e.:

$$
\mathrm{P}_{\mathrm{L}}=\frac{1000 \mathrm{ph}}{1 \mathrm{~ms}} \times \mathrm{h} v \times \frac{4 \pi \mathrm{z}^{2}}{\mathrm{~A}_{\mathrm{WFS}}} \times \frac{1}{\mathrm{X}^{2} \mathrm{~N} \sigma}
$$

For the values listed in Table 1, PL @ $1000 \mathrm{~W}$.

The pulse duration of the AVLIS laser is a few $\tau_{\mathrm{n}}$ and a simple rate equation approach argument is sufficient to highlight the issue of saturation of the sodium layer. If the peak power at the sodium layer exceeds the saturation value, $I_{s a t}=h v / \sigma \tau_{v}$, the radiation switches from the spontaneous isotropic mode to stimulated emission, and the re-radiation is in a dominantly forward direction, away from the groundbased detector. The efficiency of creating sodium backscatter therefore drops. Thus the pulse duration $\tau_{p}$ should satisfy the relation:

$$
\tau_{\mathrm{p}} / \tau_{\mathrm{n}} \geq\left(\mathrm{P}_{\mathrm{L}} / \mathrm{PRF}\right)(\sigma / \mathrm{h} v) \mathrm{A}_{\mathrm{s}}{ }^{-1} \geq\left(\mathrm{P}_{\mathrm{L}} / \mathrm{PRF}\right)(\sigma / \mathrm{h} v) \mathrm{r}_{\mathrm{O}}{ }^{2} /(\lambda \mathrm{z})^{2}
$$

where $\mathrm{PL}_{\mathrm{L}}$ is the average power of the laser, $\mathrm{PRF}$ is the laser's pulse repetition frequency, $h v$ is the photon energy at $589 \mathrm{~nm}$, and $\lambda$ is the wavelength. The second relation arises because the spot diameter at the sodium layer should be about equal to the resolution limit for a subaperture of diameter $r_{0}$. The I.LNL approach uses a high PRF laser at $26 \mathrm{kHz}$, with pulse duration of $32 \mathrm{nsec}$. Equation 3 then shows that in this case the maximum average laser power which avoids saturation is approximately $130 \mathrm{~W}$. Hence, in order to avoid saturation with the $1100 \mathrm{~W}$ LLNL laser system, the pulse length should be stretched by at least a factor of $1100 / 130=8.5$. More detailed considerations which include accounting for the wings of the pulse shape will modify the details of this pulse-stretching requirement, but the general point is clear: to avoid saturation and obtain the highest signal return with the AVLIS laser at $1100 \mathrm{~W}$ average power, the laser pulse length should be stretched by at least a factor of 10 .

The spectral profile of the sodium $\mathrm{D}_{2}$ line complex at the $200 \mathrm{~K}$ temperature in the sodium layer consists of two Doppler broadened lines each about a gigahertz wide, whose line centers are separated by $1.7 \mathrm{GHz}$. For optimum sodium excitation and re-emission efficiency, the width of the laser line profile should be comparable to $3 \mathrm{GHz}$. The transform limited pulse duration for such a spectral profile is a few tenths of a nanosecond, which is effective providing many such pulses are stacked to satisfy the pulse duration requirement discussed above. A mode locked train with such characteristics is used by Lincoln Laboratory and is an acceptable format. For longer laser pulses such as we use, the transform limited spectral profile is narrower than the sodium line-shape and phase modulation must be used to broaden the bandwidth for efficient operation. Phase modulation can be tailored to produce a spectral profile which matches the double peaked sodium distribution, thus increasing the effective hackscatter cross section.

For optimum performance from a Shack-Hartmann wave front sensor, the spot diameter at the sodium laver must be comparable to the resolution limit of a subaperture with diameter $r_{0}$. To accomplish this with the smallest beam director, a flat wavefront is needed. According to the theory of beam propagation in turbulent atmospheres, a beam of diameter $r_{0}$ with flat wavefrom will not be broadened significantly beyond its diffraction limit but will wander in position at the sodium layer, relative to the background stars. This uncertainty in the position of the heacon is well known. and astronomical laser guide star adaptive optics systems must use a natural guide star to correct for this tip-tilt effect of the at mosphere. If the laser's outgoing wavefront is not perfecty flat, the spot will he larger as well as having an unpredictable position. The spot can be made smaller by increasing the diameter of the beam an the beam direcers and this remedy

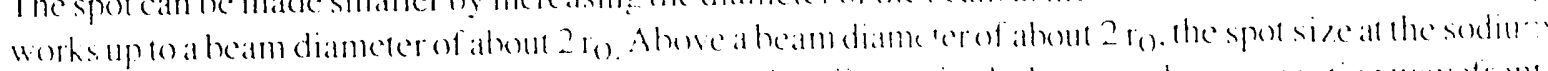

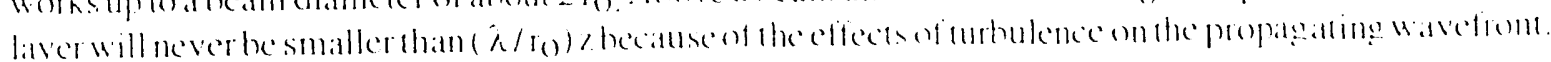


Thus, laser beam qualities of about $2 \times$ the diffraction limit can be tolerated at the expense of a larger beam director, but beyond that figure, the system performance will suffer.

\section{The AVLIS Laser System}

The AVLIS laser system presently delivers over a kilowatt of average power tuned to $589 \mathrm{~nm}$ at a PRF of $26 \mathrm{kHz}$. The bandwidth of the unstretched laser pulse is of the order of $50 \mathrm{MHz}$ which is near the transform limit of the 32 ns (FWHM) pulse duration. The center frequency is stabilized to a few tens of megahertz for indefinite periods and using phase modulation, a spectral profile is broadened and shaped to closely match that of the mesospheric sodium atoms. The laser system contains closed-loop control systems to (1) point and track the beam, (2) correct the wavefront at low spatial and temporal frequencies, and (3) remove high speed jitter. Using these control loops within the laser, beams of $1400 \mathrm{~W}$ at $589 \mathrm{~nm}$ with corrected wavefronts (RMS wavefront error $\leq 1 / 30$ wave) and stabilized jitter (RMS jitter $\leq 5 \mu \mathrm{rad}$ ) have been routinely delivered. With transmission losses, we currently transmit $1100 \mathrm{~W}$ into the atmosphere.

The AVLIS laser system is shown schematically in Fig. 3. It consists of twelve chains of copper vapor lasers (CVL's) pumping three chains of dye lasers. For the laser guide star application, one of the dye laser chains is tuned to the sodium wavelength and diverted underground to the telescope site located $100 \mathrm{~m}$ from the laser building. The output of the twelve-laser CVL system routinely exceeds $9000 \mathrm{~W}$ and the dye laser system delivers over $2 \mathrm{~kW}$ of tuned light with over a kilowatt in the highest power chain.

\section{Pulse Stretcher}

To operate the AVLIS laser at the $1100 \mathrm{~W}$ power level and avoid saturation, a multi-pass optical system consisting of multiple White cells has been designed and is currently undergoing laser tests in the laboratory. The concept consists of a series of four optical delay lines and $50 / 50$ beam splitters which create a series of 16 sub-pulses stacked head-to-tail for each input pulse. Each sub-pulse in the stack is of equal magnitude, neglecting mirror losses, so that the net effect is to lower the peak power by a factor of 16 . The pulse stretcher will be used in the fall of 1993 to increase the signal levels but all results shown in this report are with unstretched pulses. At astronomical sites, where the seeing is better and the laser power requirements lower, pulse stretching should not be required.

\section{Beam Propagation and Photometry of the Laser Guide Star}

The first laser beam propagation occurred on July 15,1992 . The event was highly visible as shown in Fig. 4. Figure 5 is a photograph of the guide star taken at a distance of four miles from the LLNL site. The length of the streak corresponds to the published thickness of the sodium layer of approximately $10 \mathrm{~km}$. Figure 6 shows a visualization of data from a CCD image of the Rayleigh scattered light (right) and the sodium-layer laser guide star (round peak towards the center), taken with a laser power of $32 \mathrm{~W}$. At this low power, the laser guide star is well separated from the lower-altitude Rayleigh-scattered light, and has a full width at half maximum of approximately 5 are seconds, corresponding to a laser irradiance spot size of roughly $2 \mathrm{~m}$ at the sodium layer. For comparison, the diffraction limited beam spread for the $4 \mathrm{~cm}$ beam dimension is $\lambda / d=1.3 \mathrm{~m}$ at the sodium layer. The secing measured on a natural star near the time this image was obrained was 3.3 are seconds. 


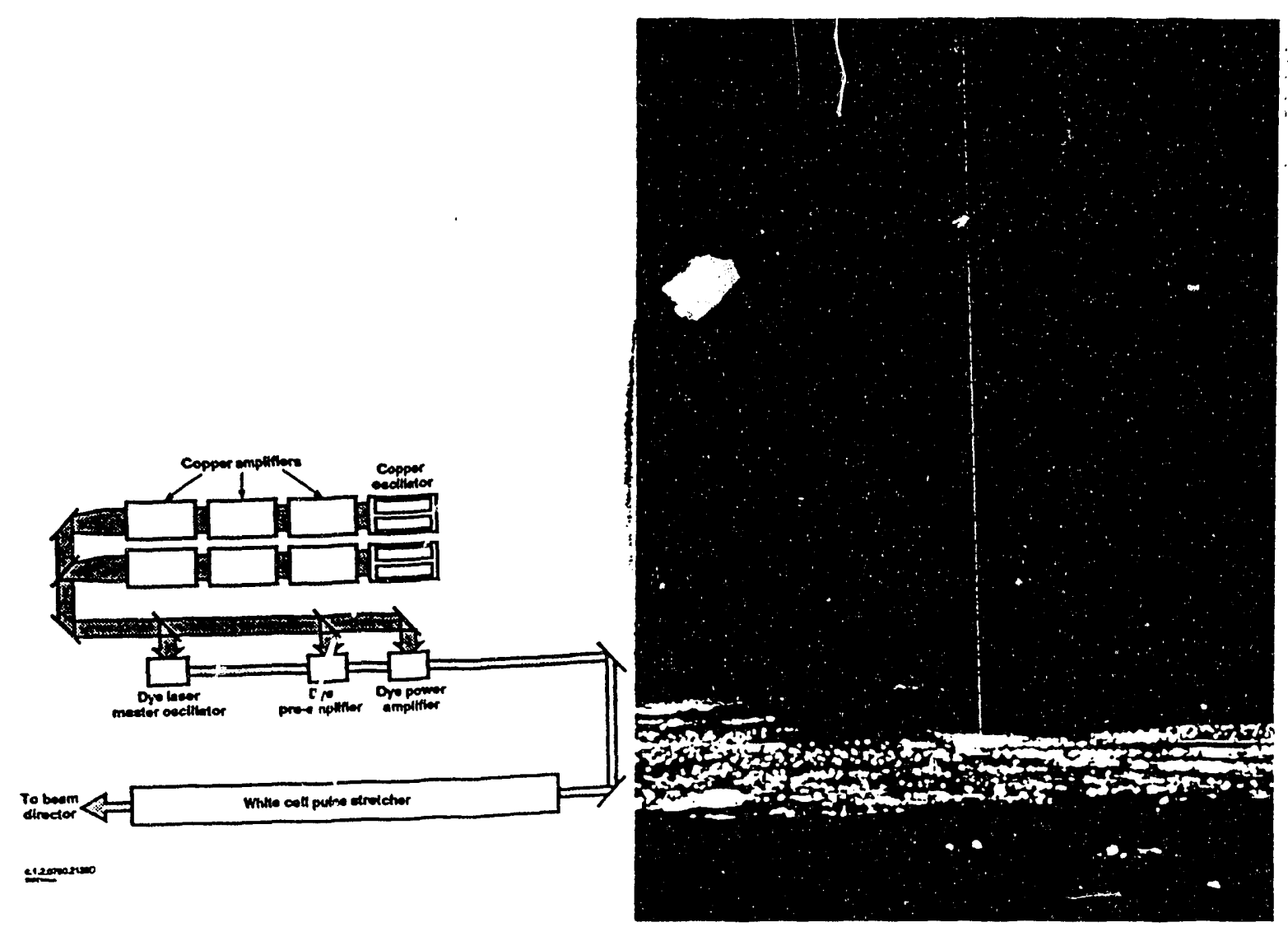

Fig. 3. Design of AVLIS laser system.

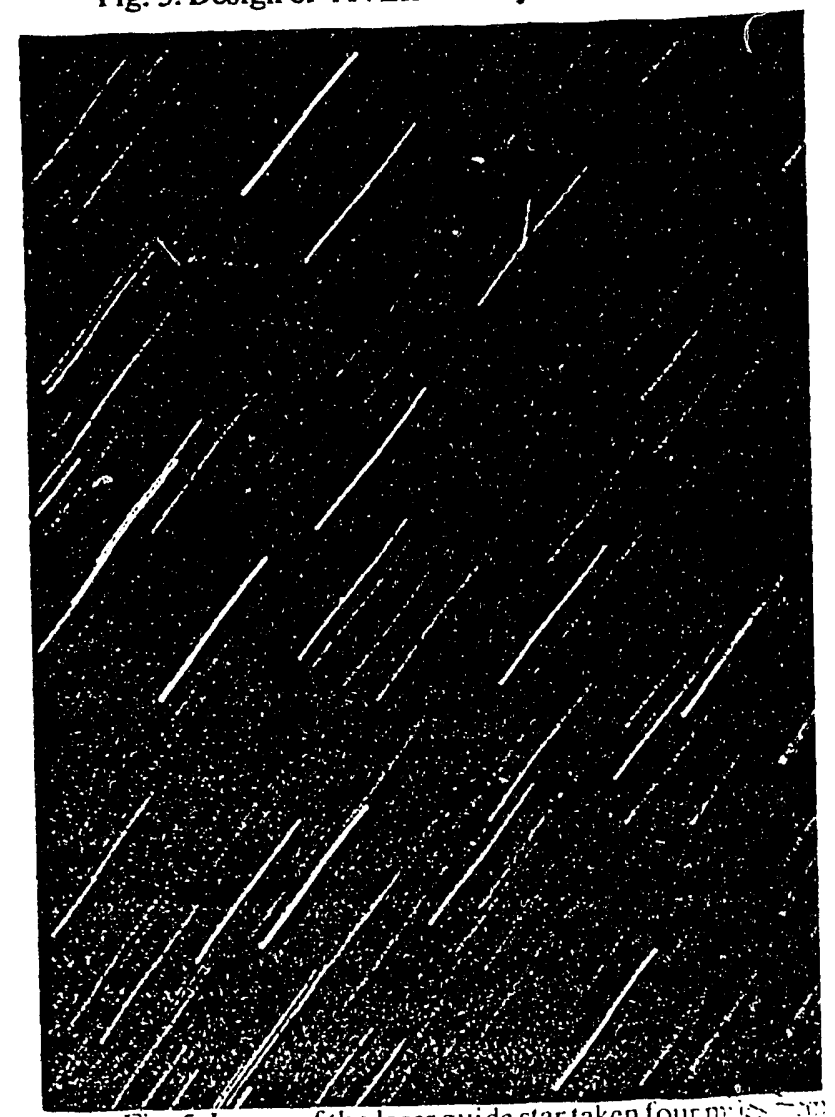

Fig 5 Image of the laser guide star taken four m:the I.LNI site.

Fig. 4. Photograph of the laser beam overlooking Livermore Valley taken from a nearby ridge.

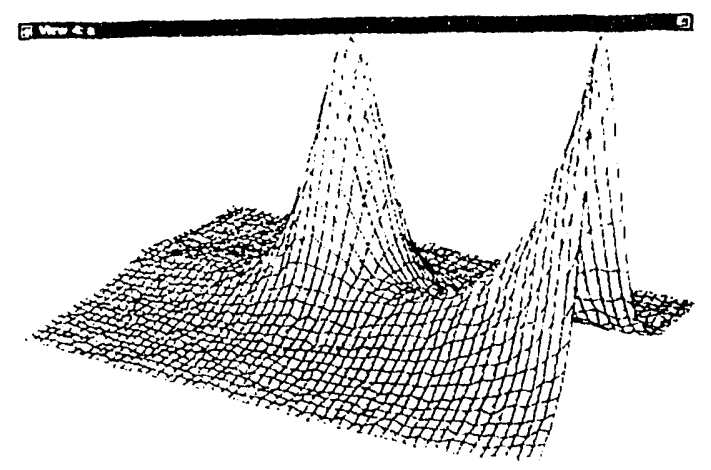

Fin. 6. Visualization of the Rayleigh scattered light (right) and sodium laserengide star (towards the center of the frame) 
At higher laser powers, the width of the sodium guide star increased, reaching a FWHM of about 7 arc seconds at a power of $1100 \mathrm{~W}$. We attribute this effect to the saturation of the sodium transition; as the laser power is raised, even the wings of the incident laser intensity distribution become saturated at the sodium layer. Computational models show that for a constant laser irradiance spot size of $2 \mathrm{~m}$ FWHM incident on the sodium layer, the guide star emission should indeed grow in diameter as the laser power is increased.

Figure 7 shows the photon flux from the sodium-layer laser guide star at the telescope aperture, as a function of the broadcast laser power. The photometric standard star Vega ( $\alpha$ Lyr) was used as a reference, with a bandpass filter centered at $550 \mathrm{~nm}$ and a bandwidth of $40 \mathrm{~nm}$ FWHM. The solid curve represents the predictions of a detailed 24-level Bloch equation model for these conditions. At the $1100 \mathrm{~W}$ level the guide star is about fifth magnitude $\left(\mathrm{m}_{\mathrm{v}}\right)$, consistent with many reports from visual observers that the guide star could be seen with the naked eye once one was 30-50 $\mathrm{m}$ away from the beam director.

\section{Open-Loop Wavefront Sensor Measurements}

We used a Shack-Hartmann wavefront sensor with the lenslet spacing corresponding to $8.4 \mathrm{~cm}$. The data in Fig. 8 shows an example of the power spectrum of subaperture tilts as a function of temporal frequency, for the laser guide star $m_{v}=5.1$ ) and a natural star, Cappella, $m_{V}=0.1$. These spectra were obtained by averaging Fourier transforms of the tilts over all of the unvignetted subapertures of the Hartmann sensor. At low frequencies, both power spectra show approximately the $v^{-2 / 3}$ ccaling predicted for Kolmogorov turbulence. The data for the natural star have sufficient dynamic range to also see the break in slone at about $20 \mathrm{~Hz}$ due to the finite size of the subapertures. In the region $20 \mathrm{~Hz}<v<100 \mathrm{~Hz}$, the scaling is approximately $v^{-8 / 3}$ in agreement with several other researchers.

The laser guide star data only extend to $60 \mathrm{~Hz}$, consistent with the use of a slower sample rate necessitated by the lower laser signal level. Nevertheless, even using the data up to $60 \mathrm{~Hz}$, it appears that the noise floor for the laser guide star measurement is a little higher than that for the natural star, as expected, because of the laser star's lower signal-to-noise ratio and farger spot size.

We have used the measured wavefront slope data to reconstruct the wavefront phase. To our knowledge this is the first time that a sodium-layer laser guide star has been used for a wavefront reconstruction. Figure 9 compares three consecutive reconstructed laser guide star wavefronts (bottom row of three frames) to three reconstructed wavefronts from a natural star at the same $8 \mathrm{msec}$ sample interval (top row of three

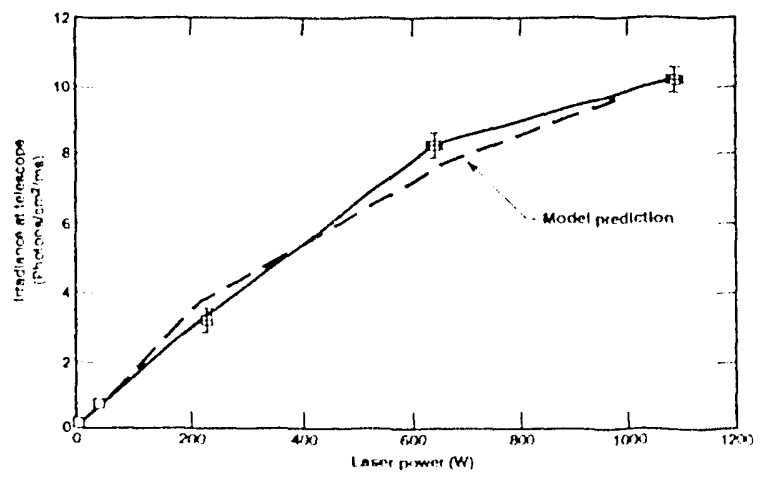

1.1:! 7. Pheson flux observed at the apenatre of the half mexterescopes

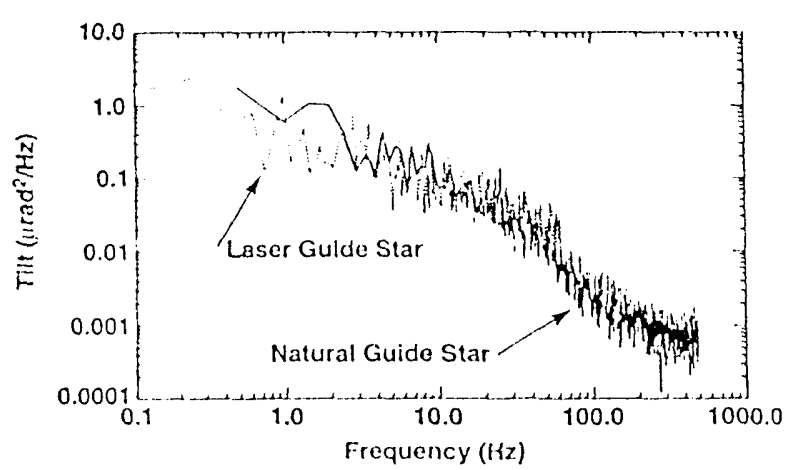

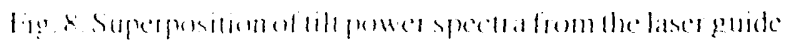

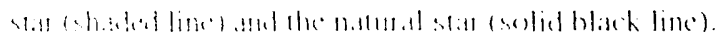



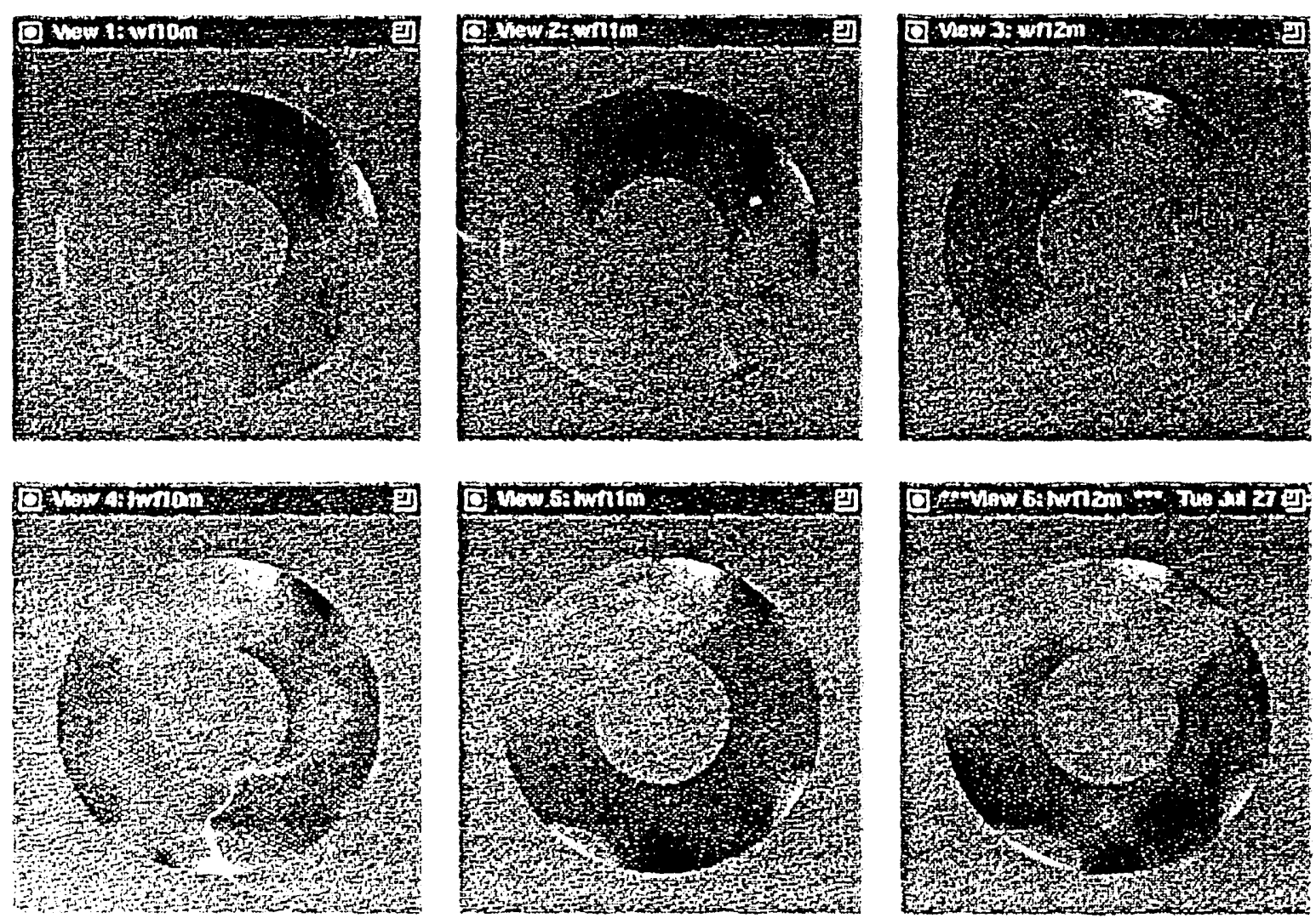

[1]

Wa

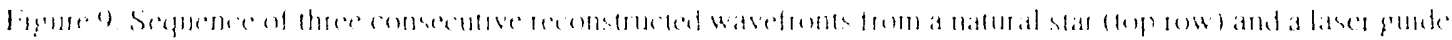
sl.m (h)

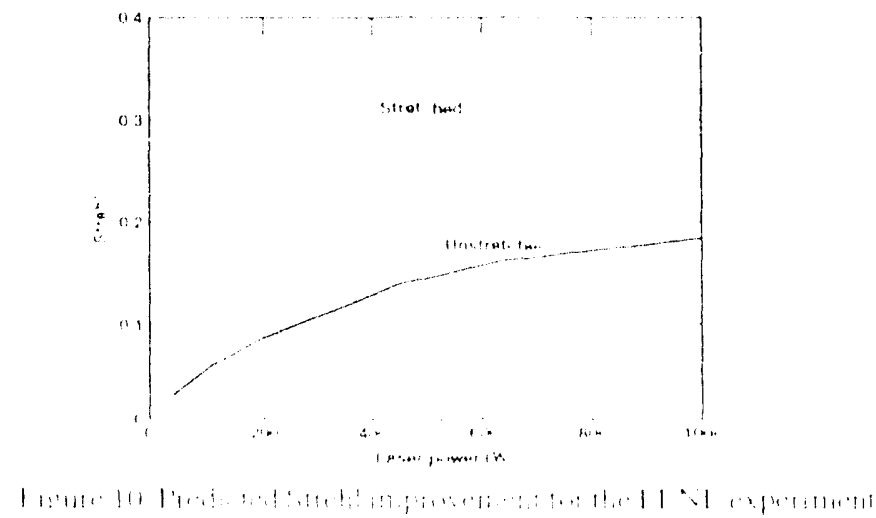

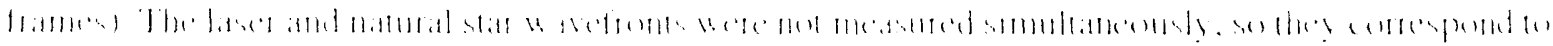

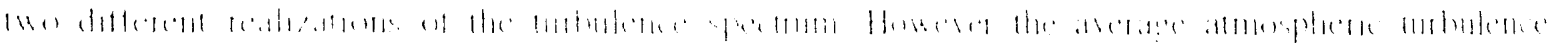

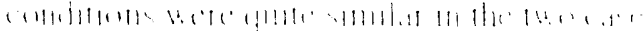

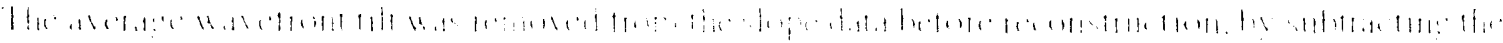

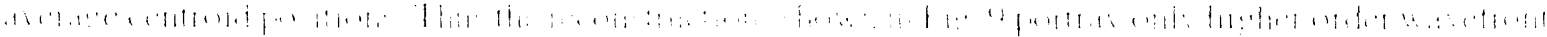


errors. Qualitatively the spatial and temporal scales of the wavefront distortions are similar for the laser guide star (bottom row) and the natural star (top row) although, of course, since the measurements werc not simultaneous, the details of the wavefronts are quite different.

\section{Implications for Closed-Loop Adaptive Optics Performance}

The long-term goal of our experiment is to demonstrate the feasibility of closed-loop adaptive optics using sodiu.n layer laser guide stars. We have used the photometry and open-loop wavefront sensor data presented to predict the performance of our planned closed-loop system using a model of the expected wavefront errors derived from statistical expressions for all the root-mean-square errors.

Figure 10 shows the Strehl ratio expected for closed-loop operation using the sodium laser guide star, as a function of the laser power, at an observing wavelength of 0.8 microns. Strehl ratios for both stretched and unstretche $\mathrm{d}$ laser pulses are shown. The wavefront sensor is assumed to be a Hartmann sensor based on our Kodak intensified motion analysis camera, better results are predicted for a low noise, bare CCD detector. The Greenwood frequency, $\mathrm{f}_{\mathrm{g}}$, was assumed to be $38.2 \mathrm{~Hz}$. At the $600 \mathrm{~W}$ power level, Strehls of about 0.3 are predicted at an observing wavelength of $\lambda=0.8 \mu$ for the stretched laser pulse. At the same ldser power, Strehls of about 0.15 at $\lambda=0.8 \mu$ are predicted for the unstretched pulse. Thus, even for unstretched laser pulses, the Strehl should be high enough with $600 \mathrm{~W}$ of laser power to distinguish a diffraction-limited core in the point-spread function, for observations of objects with a sufficientiy high signal to noise ratio.

\section{ACKNOWLEDGMENTS}

This research was performed under the auspices of the U.S. Department of Energy, under contract number W-7405-ENG-48 to the Lawrence Livermore National Laboratory. The LLNL Laser Guide Star Project is supported by the LLNL Laboratory Directed Research and Development Program. Related work is supported by the Institute of Geophysics and Planetary Physics and by the Engineering Department at LLNL.

The author would like to thank C. Max, K. Avicola, J. Brase, H. Bissinger, J. Duff, D. Gavel, J. Horton, J. Morris, S. Olivier, R. Presta, D. Rapp, T. Salmon. D. Smauley and K. Waltjen, who comprise the Laser Guide Star Team at LLNL. These people performed much of the work presented in this paper.

\section{REFERENCES}

1. W. Happer, G. MacDonald, C. Max, and F. Dyson, "Amospheric Turbulence Compensation Using Resonant Optical Backscattering from the Sodium Layer in the Upper Amosphere", paper aceepted for this Feature Issue of J.O.S.A.

2. R. Foy and A. Labeyric, "Feasibility of Adaptive Optics Telescope with Laser Probe", Astronomy" and Astrophysics 152, L29-L.31 (1985); L. A. Thompson and (. S. Gardner, "Experiments on Laser Guide Stars at Matma Kea Observatory for Adaptive Imaging in Astronomy," Nature 328, 229 - 231 (1987); R. A. Humphries, ('. Primmerman, L. Bradley and I. Hermann., "Atmospheric Turbulence

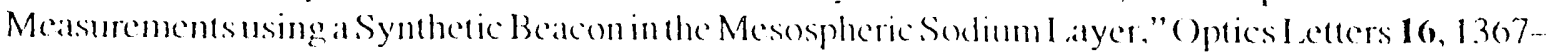
$1369(1991)$. 

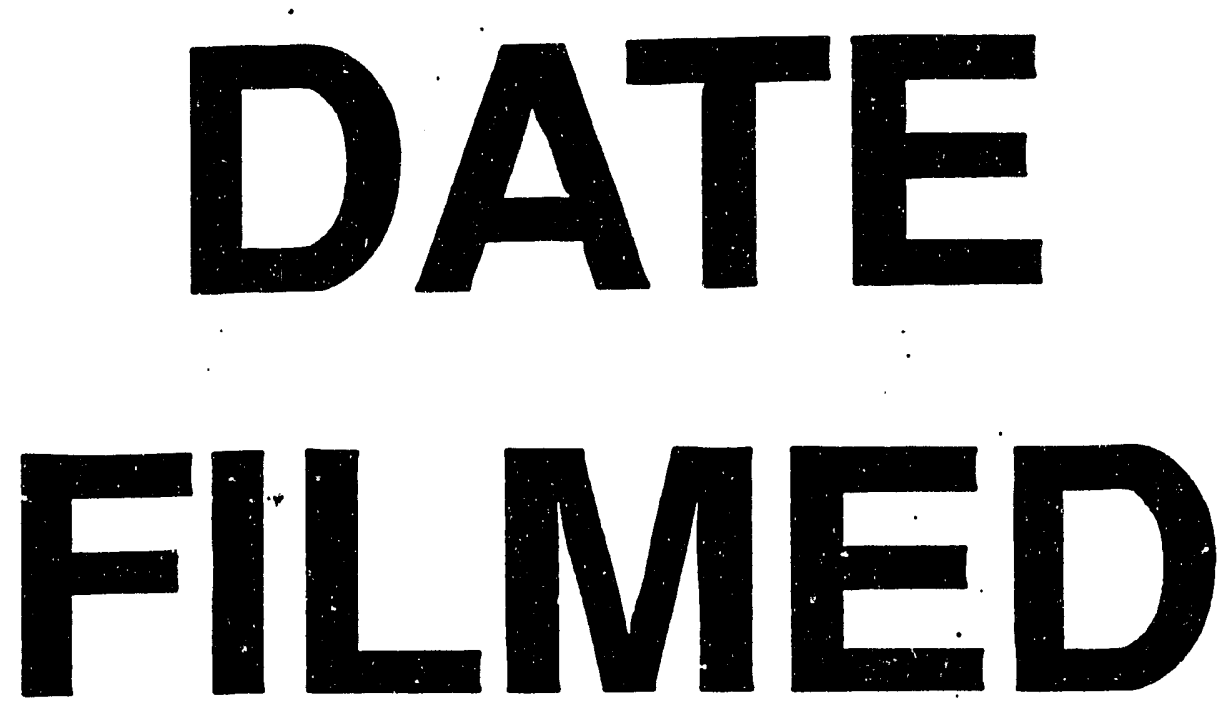

$12 / 14 / 93$
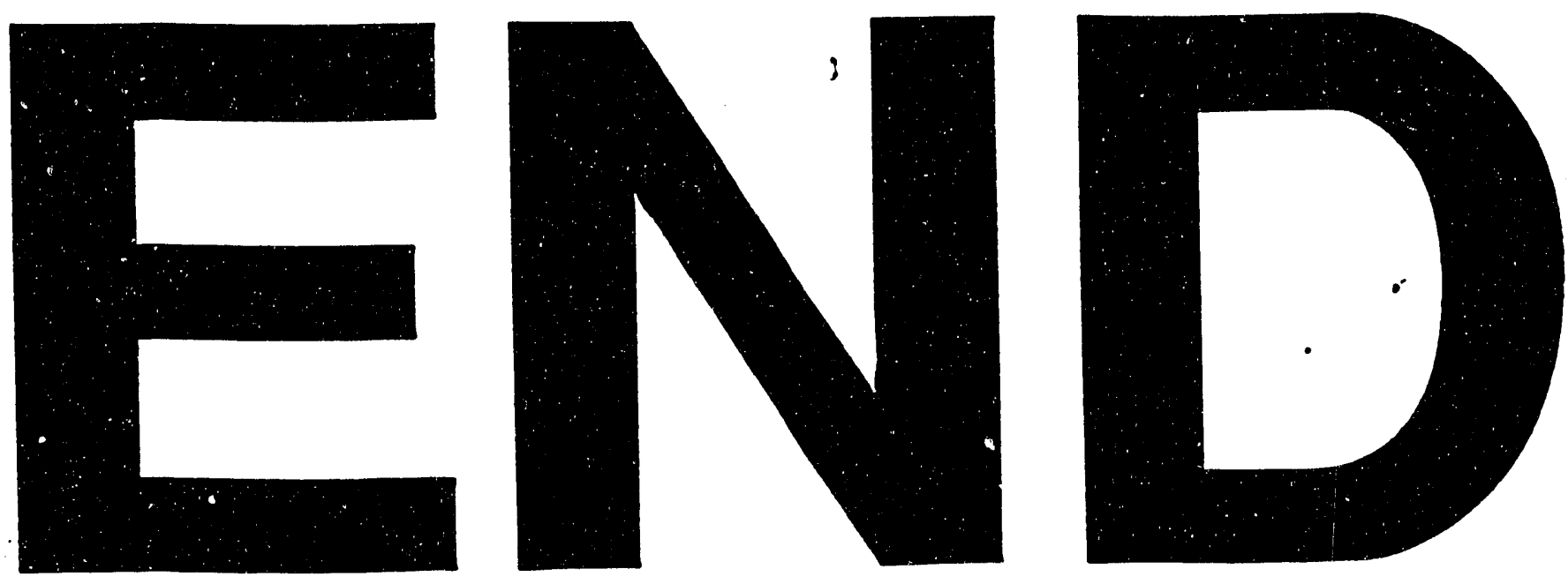


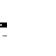

